\title{
PILOT: RCT OF ADJUNCTS TO GENERAL ANESTHESIA IN PEDIATRIC DENTISTRY
}

William PS McKay MD, Kirstin Derdall BSc., Department of Anesthesia, University of Saskatchewan, RUH, 103 Hospital Drive, Saskatoon SK, S7N 0W8

INTRODUCTION Small children with extensive caries require dentistry under general anesthesia (GA). Their course is often marred by vomiting $(35 \%)^{1}$, prolonged crying, and emergence agitation (EA, 18\% $)^{2}$. Rapid awakening, pain, inflammation, and nausea are suspected causes. Adjuncts to GA used to prevent these problems have not been systematically studied. A randomised controlled trial (RCT), of intravenous (IV) morphine, dexamethasone, and granisetron, was used to study effects on emergence crying, vomiting, EA, and wellness at next-day followup.

METHODS With Research Ethics Board approval, 100 healthy subjects were randomised with full blinding and concealment. All had oral ibuprofen $10 \mathrm{mg} / \mathrm{kg}$, then a standard GA: mask induction halothane, an IV infusion of saline; $2 \mathrm{mg} / \mathrm{kg}$ of propofol, then intubation; maintenance with halothane and nitrous oxide. Study drug: saline placebo (group 0), morphine $50 \mathrm{ug} / \mathrm{kg}$ (group 1), dexamethasone 150ug/kg (group 2), granisetron 40ug/kg (group 3), or a combination of active drugs in half doses (group 4).

\section{RESULTS}

\begin{tabular}{|l|l|l|l|l|}
\hline & Group differences & Mean (SD) & Mean (SD) & P \\
\hline Age & None & & & 0.63 \\
\hline Weight & None & & & 0.65 \\
\hline Awakening time & None & & & 0.67 \\
\hline $\begin{array}{l}\text { Loud crying } \\
\text { Duration (min) }\end{array}$ & $\begin{array}{l}\mathbf{1} \mathbf{4} \\
\text { Rest not significant }\end{array}$ & $1.00(2.15)$ & $10.000(11.64)$ & 0.04 \\
\hline Vomiting PACU & No & & & 0.06 \\
\hline EA & $\mathbf{1}$ better than others & & & 0.006 \\
\hline $\begin{array}{l}\text { Vomiting later } \\
\text { (episodes) }\end{array}$ & $\begin{array}{l}\text { 1 worse than others } \\
\text { (placebo best) }\end{array}$ & $2.42(2.43)$ & $0.08(0.28)$ & $<0.0001$ \\
\hline Wellness next day & None & & & 0.497 \\
\hline
\end{tabular}

DISCUSSION Morphine prevented EA and crying in PACU, but caused more vomiting after leaving the clinic. Numbers are insufficient to show other adjuncts were ineffectual.

REFERENCES 1. Davis PJ, McGowan FX, et al. Anesthesiology 1995; 83: 956 - 960.

2. Voepel-Lewis T, Malviya S, Tait AR. Anesth Analg. 2003 Jun; 96(6): 1625-30 